\author{
Iván Durán-Sáenz",* \\ Susana Espinosa-Villar² \\ Dorleta Martín-Diez² \\ Irene Martín-Diez ${ }^{2}$ \\ Ruth Martínez-Valle ${ }^{2}$ \\ Ana Elisa del Val-Labaca²
}

1. Escuela Universitaria de Enfermería. Osakidetza-Servicio Vasco de Salud. Vitoria-Gasteiz. País Vasco. España.

2. Hospital Universitario de Araba. Osakidetza-Servicio Vasco de Salud. OSI Araba. Vitoria-Gasteiz. País Vasco. España.

*Autor para correspondencia.

Correo electrónico: ivan_duran001@ehu.eus (Iván Durán-Sáenz).

Recibido el 27 de noviembre de 2019; aceptado el 17 de enero de 2020.

\section{Conocimiento, actitud y práctica sobre pie diabético en pacientes o sus cuidadores en cirugía vascular}

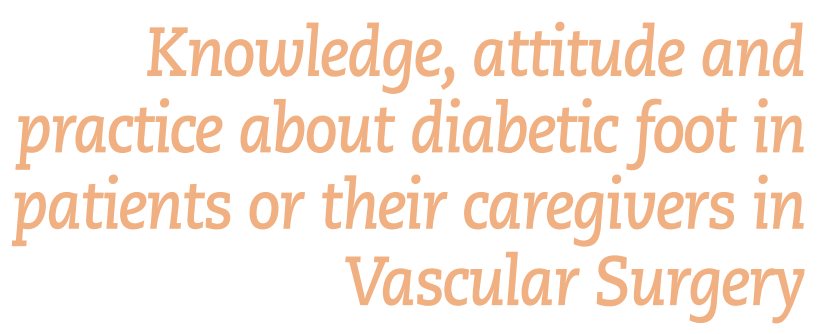

\section{RESUMEN}

Objetivos: Identificar el nivel de conocimiento, actitud y práctica orientada a la prevención del pie diabético en personas ingresadas o sus cuidadores principales en la Unidad de Cirugía Vascular. Metodología:

Se realizó un análisis estadístico descriptivo transversal. Las variables analizadas fueron: conocimiento, actitud y comportamientos relacionados con el cuidado del pie diabético. Desde diciembre de 2016 a diciembre de 2017, un equipo de enfermeras facilitó un cuestionario durante el ingreso de los pacientes en la Unidad de Cirugía Vascular, el cual no se había utilizado anteriormente con población española. Resultados: El tamaño de la muestra se calculó con el objetivo de conseguir 189 personas. Un total de 172 respondió el cuestionario. En la dimensión de conocimiento,

la media fue de 6,56 sobre $10(\mathrm{DE}=2,05)$. El nivel de conocimiento de un $58,2 \%$ fue medio. En la dimensión de actitud, la media fue de 4,42 sobre $5(\mathrm{DE}=0,95)$. Un 93\% declaró tener actitud favorable hacia el autocuidado de sus pies. En la dimensión de práctica orientada a la prevención, la media fue de 5,45 $(\mathrm{DE}=2,55)$. El 51,5\% realizó una práctica media de autocuidado del pie diabético. Conclusiones: Las personas poseían conocimientos medios de los cuidados del pie diabético. Al preguntarles por su actitud, esta fue favorable. Contrasta con el nivel de comportamiento en la práctica de autocuidado, que en la mitad de la muestra fue de carácter medio, repartiendo en partes iguales la buena práctica con la escasa práctica de autocuidado.

PALABRAS CLAVE: Diabetes; pie diabético; conocimientos, actitudes y práctica en salud.

\section{ABSTRACT}

Background: To identify the level of knowledge, attitude and practice oriented to the prevention of diabetic foot in hospitalized persons or their main caregivers in the Vascular Surgery Unit. Methods: A transversal descriptive statistical analysis was carried out. The variables analyzed were: knowledge, attitude and behavior related to the care of the diabetic foot. A team of six nurses, provided a questionnaire during the period of admission of patients from December 2016 to December 2017, which had not previously been used with Spanish population. Results: The sample size was calculated in order to get 189 people. A total of 172 people answered the questionnaire. In the Knowledge Dimension, the mean was (6.56) out of 10 (SD = 2.05). The level of knowledge of $58.2 \%$ was medium. In Attitude, the average was 4.42 out of 5 $(S D=0.95) .93 \%$ say they are favorable towards self-care of their feet. In Practice oriented to prevention, the average was 5.45 (SD $=2.55$ ). $51.5 \%$ perform an average self-care practice of diabetic foot. Conclusions: The people in the study had average knowledge of diabetic foot care. When asked about their attitude, this was favorable. It contrasts with the level of behavior in the practice of self-care, which in the middle of the sample was of average character, distributing in equal parts the good practice with the scarce practice of self-care.

KEYWORDS: Diabetes; diabetic foot; Health Knowledge, Attitudes, Practice.

\section{$\searrow$ INTRODUCCIÓN}

La diabetes es una patología crónica que puede clasificarse como diabetes tipo 1 y diabetes tipo 2, diabetes mellitus gestacional o diabetes específicas producidas por otra causa ${ }^{1}$. En el año 2014 se estimó que 422 millones de personas en el mundo tenían diabetes ${ }^{2}$. La prevalencia global de diabetes en Euskadi en mayores de 18 años es del 10,6\%. En España, estudios realizados con la misma metodología y con el mismo fin observaron que la prevalencia es del 13,8\% ${ }^{3}$. Estos niveles de prevalencia la convierten en una epidemia mundial. La neuropatía diabética afecta al 50\% de las personas afectadas cuando llevan más de 15 años de evolución de la enfermedad $^{4}$. En el caso de la enfermedad vascular periférica arterial oclusiva presentan una prevalencia en torno al $25 \%$, cifra que se incrementa hasta el $49 \%$ en aquellos que presentan úlceras o lesiones en el pie 5 .

Estas afecciones facilitan la aparición de complicaciones de las diabetes tipos 1 y 2 que surgen a largo plazo; casi nunca ocurren en los primeros 5 a 
10 años tras el diagnóstico ${ }^{6}$. Una de las complicaciones que mayor impacto provoca en las personas por su repercusión en la calidad de vida y riesgo de sufrir una amputación es el pie diabético, definido por la Organización Mundial de la Salud como: "La presencia de ulceración como infección y/o gangrena en el pie asociado a neuropatía diabética y a diferentes grados de enfermedad vascular periférica como consecuencia de la interacción compleja de diferentes factores inducidos por una hiperglucemia mantenida”.

El término pie diabético hace, por tanto, referencia al síndrome resultante de la interacción de diversos factores sistémicos (angiopatía, neuropatía e infección) y factores externos ambientales (modo de vida, higiene local, calzado inadecuado) e intrínsecos ${ }^{6}$, que no solo favorecen la aparición de la úlcera, sino que también contribuyen a su desarrollo y perpetuación. La mayor prevalencia de ulceración del pie diabético se presenta en América del Norte (13,0\%) y la menor tasa se presenta en Oceanía (3,0\%). La prevalencia de la úlcera del pie diabético es relativamente más alta en África $(7,2 \%)$ que en Asia $(5,5 \%)$ y Europa $(5,1 \%)^{7}$. En Europa, la tasa anual de amputación para las personas con diabetes se ha estimado en el 0,5-0,8\%, y en los EE. UU. se ha notificado que alrededor del $85 \%$ de las amputaciones de los miembros inferiores se vieron precedidas de úlcera de pie diabético ${ }^{8}$. Según datos del estudio Eurodiale, una lesión de este tipo que no se cura en el transcurso de un año tiene un coste aproximado de $19000 €$ por paciente; cuando estas conducen a la amputación del miembro inferior, los costes se incrementan a $25000 €$ por amputación ${ }^{9}$. Respecto a las úlceras de la extremidad inferior de otras etiologías, es en las de etiología neuropática donde las medidas de prevención primaria presentan unos datos no solo de máxima eficacia, sino también de indiscutible eficiencia ${ }^{10}$.

Bakker y Riley ${ }^{11}$ afirmaron que en el tratamiento de las lesiones del pie relacionadas con la diabetes quedó demostrado que entre un $49 \%$ y un $85 \%$ de todos los problemas relacionados con el pie diabético se pueden prevenir si se adoptan las medidas apropiadas. En lo que respecta al efecto de la educación en diabetes para la prevención de la ulceración del pie diabético, una revisión Cochrane encontró nueve estudios clínicos aleatorios, de los cuales solo un estudio que incluyó pacientes de alto riesgo demostró una reducción en la incidencia de úlceras [odds ratio $(\mathrm{OR}): 0,28$, intervalo de confianza (IC) al 95\%: 0,13 a 0,59] y en la tasa de amputación (OR: 0,32, IC 95\%: 0,14 a 0,71) después de un año. La educación a corto plazo del paciente parece influir de manera positiva en el conocimiento acerca del cuidado de los pies y en el comportamiento de los pacientes ${ }^{12}$. Igualmente, la American Diabetes Association (ADA), en su Standards of Medical Care in Diabetes, determina que la educación y el seguimiento en autocuidados son elementos esenciales en los cuidados diabéticos. Todos los pacientes con diabetes, y particularmente aquellos con condiciones de alto riesgo y sus familias o cuidadores, deben recibir educación general sobre factores de riesgo y manejo apropiado ${ }^{1}$.

Consideramos que para realizar una intervención individualizada y eficiente en educación es necesario identificar el nivel de conocimiento, actitud y comportamiento en autocuidados del pie diabético llevados a la práctica en personas diabéticas o sus cuidadores en caso de deterioro cognitivo o dependencia para las actividades básicas de la vida diaria. Teniendo en cuenta todo lo expuesto, se pretende iniciar un acercamiento a la prevención del pie diabético, comenzando por la valoración de los pacientes diabéticos/cuidadores para determinar necesidades y vacíos que requieran estrategias de intervención.

\section{Y OBJETIVO}

Identificar el nivel de conocimiento, actitud y práctica orientada a la prevención del pie diabético en personas ingresadas o sus cuidadores principales en una unidad hospitalaria de cirugía vascular.

\section{MÉTODO}

Estudio descriptivo transversal, realizado entre diciembre de 2016 y diciembre de 2017.

Se desarrolló con la participación de pacientes con diagnóstico de diabetes tipo 1 o 2 que ingresaron en la Unidad de Hospitalización de Cirugía Vascular del Hospital Universitario Araba (HUA-OSI Araba). En caso de que los pacientes presentaran deterioro cognitivo objetivado a través de cuestionario Pfeiffer ${ }^{13}$ o diagnóstico NANDA de "Déficit de autocuidado: baño o vestido ${ }^{14}$ ", tras valoración por dominios, se facilitó la encuesta a sus cuidadores principales.

Se establecieron como criterios de exclusión:

- Pacientes con deterioro cognitivo sin cuidador principal, por la imposibilidad de obtener datos.

- Pacientes institucionalizados, debido a que sus cuidados son efectuados por personal sanitario profesional.

- Pacientes con amputación de ambas extremidades inferiores.

Se calculó que el tamaño de la muestra para una proporción esperada del $40 \%$ de pacientes diabéticos con un nivel de conocimientos bueno en la dimensión de conocimientos del cuestionario KAP (Knowledge-Attitude-Practice) $(\geq 8 / 10 \text { puntos) })^{14}$, con una precisión del $7 \%$, nivel de confianza del $95 \%$ y una potencia del $80 \%$, debía ser de 189 pacientes. El cálculo muestral se llevó a cabo mediante el programa informático Epidat 3.1.

Como variables principales del estudio se incluyeron:

- Nivel de conocimientos sobre el cuidado del pie diabético.

- Nivel de actitud (autoeficacia) en el cuidado del pie diabético.

- Nivel de comportamiento en autocuidados del pie diabético llevados a la práctica.

El cuestionario KAP utilizado es un cuestionario elaborado desde las recomendaciones del American College of Foot and Ankle Surgeons y la OMS ${ }^{15}$ y ha sido empleado en estudios previos ${ }^{14,16,17}$, aunque no en población española. Para la selección, adaptación, traducción y verificación del grado de compresión se contó con la participación de una enfermera supervisora de investigación en cuidados, una enfermera supervisora y 5 enfermeras de hospitalización de la Unidad de Cirugía Vascular. El cuestionario consta de tres secciones, una de 10 preguntas sobre conocimientos, una de 5 preguntas para valorar las actitudes y otra de 8 preguntas para evaluar el comportamiento en el autocuidado del pie diabético. Cada respuesta correcta representó un punto, excepto para los dos últimos ítems de la dimensión de comportamiento, que pueden puntuar 0,1 o 2, según la opción de respuesta ${ }^{14}$. Para la dimensión de conocimiento, una puntuación igual o menor que 3 indicó que los encuestados tienen conocimientos pobres; entre 4 y 7 , conocimientos medios, e igual o superior a 8, conocimientos buenos. En la dimensión de actitud, una puntuación igual o menor que 2 mostró una actitud poco favorable al autocuidado del pie diabético e igual o superiora a 3 se consideró una actitud favorable. Respecto a la dimensión de comportamiento, una puntuación igual o inferior a 3 correspondió con quien realizaba una práctica considerada escasa de autocuidados del pie, entre 4 y 7 se asoció con quien realizó una práctica considerada como media en autocuidados del pie e igual o superior a 8 se asoció con las personas que realizaban una buena práctica de autocuidados del pie. Se tuvieron también en cuenta variables sociodemográficas y clínicas. 
El aspecto más importante recogido en la bibliografía es que el $85 \%$ de las amputaciones son evitables con un apropiado cuidado y educación ${ }^{15}$. Considerando que se puede individualizar el consejo sanitario en función del conocimiento, se recogieron los datos. Se aplicó un muestreo no probabilístico de conveniencia. Los autores del estudio fueron los encargados de identificar a pie de cama a pacientes y cuidadores informantes durante el transcurso del ingreso hospitalario. Estos fueron reconocidos como diabéticos según los criterios de la ADA mediante la entrevista realizada al ingreso hospitalario por dominios.

A lo largo del ingreso y tan pronto como fue posible se realizó una solicitud de consentimiento informado. Inmediatamente después, en caso de ser apto para el estudio, tanto a pacientes como a cuidadores se les administró el cuestionario para su autocumplimiento y se aclararon dudas en caso necesario. Una vez recogidos los cuestionarios necesarios para el estudio, se transcribieron a una base de datos para su análisis estadístico posterior.

Las variables cuantitativas se describen mediante medias y desviaciones estándar. Las variables cualitativas se describen mediante frecuencias y porcentajes y se acompañaron con su intervalo de confianza al 95\%. Se aplicó la prueba de la $\chi^{2}$ para el análisis bivariante. Se tomo un nivel de significación de 0,05 , y los datos fueron procesados con el programa estadístico SPSS v.23.

El estudio se desarrolló siguiendo los principios éticos para la investigación en seres humanos teniendo en cuenta la Declaración de Helsinki. Asimismo, se obtuvo dictamen favorable del Comité de Ética en Investigación de la OSI Araba (Expte. 2016-080). Todos los encuestados fueron informados verbalmente del proyecto y otorgaron su consentimiento plasmando su firma en el documento desarrollado para tal fin. Durante todo el proceso, la privacidad y la protección de datos estuvieron garantizadas, respetándose lo estipulado en la LOPD 15/1999 y los derechos ARCO. Durante el proceso, el Cuaderno de Recogida de Datos no albergó ningún dato identificativo de la persona encuestada.

\section{Y RESULTADOS}

Finalmente, participaron en el estudio un total de 172 personas. Las características de la muestra se pueden ver en la tabla 1 . Fueron cumplimentadas 32 encuestas por cuidadores principales, las cuales se incluyeron junto al resto de los cuestionarios de personas con diabetes, al ser estos los responsables directos del conocimiento, actitud y comportamiento en autocuidados del pie diabético llevados a la práctica. De los cuestionarios cumplimentados por cuidadores principales, 14 correspondieron al criterio de deterioro cognitivo y 18 a déficit de autocuidado.

\section{Nivel de conocimientos sobre el cuidado del pie diabético}

El 58,2\% ( $\mathrm{n}=99)$ de los participantes presentaron un nivel de conocimiento medio sobre el pie diabético según la correlación de respuestas correctas citada anteriormente. El 34,7\% ( $\mathrm{n}=59)$ tuvo conocimientos buenos y un 7,1\% $(n=12)$, pobres. La media en esta variable fue de 6,56 respuestas correctas sobre 10 ítems $(\mathrm{DE}=2,05)$.

\section{Nivel de actitud (autoeficacia) en el cuidado del pie diabético}

En cuanto a la valoración de la dimensión de actitud, un 93\% ( $\mathrm{n}=159)$ de la muestra declaró tenerla favorable hacia el autocuidado de sus pies.
Tabla 1. Características de la muestra $(n=172)$

\begin{tabular}{|l|l|r|}
\hline Variables & 30-69 años & $77(44,8)$ \\
\hline \multirow{2}{*}{ Edad } & $>70$ años & $95(55,2)$ \\
\hline \multirow{2}{*}{ Género } & Masculino & $133(77,3)$ \\
\hline & Femenino & $39(22,7)$ \\
\hline & Sin estudios & $28(16,3)$ \\
\hline Nivel de estudios & Primaria & $88(51,2)$ \\
\hline & Secundaria/Medios & $33(19,2)$ \\
\hline Deterioro cognitivo & Superiores & $23(13,4)$ \\
\hline Cuidador principal & Sí & $14(8,1)$ \\
\hline Tiempo de evolución de la diabetes: & $<15$ años & $32(18,6)$ \\
\hline fecha de diagnóstico (año) & $\geq 15$ años & $84(48,8)$ \\
\hline \multirow{2}{*}{ Presencia de lesiones en los pies } & No & $88(51,2)$ \\
\hline & Sí & $60(34,9)$ \\
\hline Hemoglobina glicosilada HbA1c & $<6,4 \%$ & $112(65,1)$ \\
\hline & $6,5-7 \%$ & $49(28,5)$ \\
\hline & $>7 \%$ & $33(19,2)$ \\
\hline
\end{tabular}

En este caso, la media fue de 4,42 respuestas correctas sobre 5 ítems $(\mathrm{DE}=0,95)$.

\section{Nivel de comportamiento en autocuidados del pie diabético llevados a la práctica}

En el comportamiento entendido como práctica en el autocuidado del pie diabético orientada a la prevención de eventos adversos encontramos que el $51,5 \%(\mathrm{n}=88)$ realizó una práctica media de autocuidado del pie diabético. El $24 \%(\mathrm{n}=41)$ realizó una práctica considerada como escasa, y el $24,6 \%(n=42)$ restante realizó una práctica buena de autocuidado. La media fue de 5,45 respuestas correctas sobre 10 ítems $(\mathrm{DE}=2.55)$. Esta dimensión se valora a través de cómo se realizan cuidados básicos y con qué frecuencia se consulta a personal sanitario.

Las respuestas de los participantes a cada ítem del cuestionario se muestran en la tabla 2.

Se observó una relación estadísticamente significativa $(p=0,045)$ entre la edad de los participantes y la actitud en el autocuidado. Sin embargo, tanto los que son mayores de 70 ańos como menores de esta edad tienen más actitud favorable que poco favorable. Con el resto de las variables no se llegó a significación estadística (tabla 3).

Un total de 112 personas del total de la muestra presentaban lesiones en sus pies. Se observó una relación estadísticamente significativa entre los niveles de HbAlc y la presencia de lesiones $(p=0,022)$. Se ha visto que de las personas con niveles de HbAlc > 7\%, el 72,2\% $(n=65)$ presentaba lesiones frente al 27,8\% $(n=25)$ que no las presentaba. De la muestra con niveles de HbAlc entre 6,5\% y 7\%, el $45,5 \%(\mathrm{n}=15)$ presentaba lesiones frente al 54,5\% $(\mathrm{n}=18)$ que no lo hacía. Se observó en la muestra con niveles de $\mathrm{HbAlc}<6,4 \%$, que el $65,3 \%(\mathrm{n}=32)$ presentaba lesiones frente al $34,7 \%(\mathrm{n}=17)$ que no las presentaba (fig. 1). 
Tabla 2. Conocimiento, actitud y comportamiento en el autocuidado del pie diabético

Valoración de la dimensión de conocimiento (puntuación total: 10)
1. ¿Es verdad que todos los pacientes con diabetes desarrollan una disminución del flujo sanguíneo
en sus pies?
2. ¿Es verdad que todos los pacientes con diabetes desarrollan una falta de sensibilidad en sus pies?
3. ¿Es verdad que todos los pacientes con diabetes desarrollan úlceras en sus pies?
4. ¿Es verdad que todos los pacientes con diabetes desarrollan gangrena?
5. ¿Ha recibido alguna información sobre el cuidado del pie diabético? Si es sí, ¿cuándo?
6. ¿Tiene conocimiento de que fumar puede reducir el flujo sanguíneo en los pies?
7. ¿Sabía que si pierde la sensibilidad de los pies es más propenso a tener úlceras de pie diabético?
8. ¿Sabía que si tiene el flujo sanguíneo disminuido en el pie es más propenso a tener úlceras de
pie diabético?
9. ¿Sabía que si tiene infección en el pie puede desarrollar heridas?
10. ¿Cuál cree que es la forma más adecuada de cortarse las uñas?

\section{Valoración de la dimensión de comportamientos en autocuidados (puntuación total: 10)}

1. ¿Está dispuesto a cambiar sus hábitos con la comida y hacer ejercicio regularmente para prevenir futuras complicaciones derivadas de la diabetes?

\begin{tabular}{|c|c|}
\hline $\begin{array}{c}\text { Sí } \\
n(\%)\end{array}$ & $\begin{array}{c}\text { No } \\
n(\%)\end{array}$ \\
\hline $50(29,2)$ & $121(70,8)$ \\
\hline $66(38,6)$ & $105(61,4)$ \\
\hline $93(54,1)$ & $79(45,9)$ \\
\hline $120(69,8)$ & $52(30,2)$ \\
\hline $76(44,2)$ & $96(55,8)$ \\
\hline $138(80,2)$ & $34(19,8)$ \\
\hline $146(84,9)$ & $26(15,1)$ \\
\hline $152(88,4)$ & $20(11,6)$ \\
\hline
\end{tabular}

2. ¿Cree que las personas con diabetes deberían adquirir la responsabilidad de examinarse sus propios pies?

3. ¿Está dispuesto a utilizar calzado especial recomendado por el podólogo/enfermera/médico?

4. ¿Utilizaría el calzado para interiores aconsejado por el podólogo/enfermera/médico?

$153(89)$

$19(11)$

Rectas

Redondeadas, cortando bordes

5. ¿Cree que puede llevar una vida normal si sigue las recomendaciones adecuadas para la diabetes?

Valoración de la dimensión de comportamientos en autocuidados (puntuación total: 10)

1. ¿Realiza la higiene de sus pies diariamente?

2. ¿Hidrata las zonas secas de sus pies diariamente?

105 (61)

67 (39)

Sí

No

$147(86)$

$24(14)$

$159(92,2)$

$13(7,6)$

3. ¿Revisa sus pies diariamente en busca de alguna herida?

$154(89,5)$

$18(10,5)$

$145(84,3)$

$27(15,7)$

$154(89,5)$

$18(10,5)$

4. ¿Qué haría si se encuentra algo anormal en sus pies? ¿Lo resolvería por sí mismo/consultaría al podólogo/enfermera/médico?

Sí

105 (61)

$84(48,8)$

89 (52)

Consultar

$121(70,3)$

$125(72,7)$

$112(65,1)$

Cuando está

dañado

$90(52,3)$

Solo cuando hay

un problema

$86(50)$

\section{No}

67 (39)

$88(51,2)$

82 (48)

Resolverlo por mí mismo

$51(29,7)$

$47(27,3)$

$60(34,9)$

Una vez al año Más de una vez

$22(12,8)$

Una vez al año

$12(7)$ al año

$60(34,9)$

Una vez cada

6 meses o más

74 (43)

\section{У DISCUSIÓN}

Este trabajo revela que el nivel de conocimiento sobre el pie diabético de los encuestados alcanza niveles medios y buenos. Estos resultados no coinciden con los encontrados en la bibliografía ${ }^{16}$, donde la muestra presenta conocimientos mayoritariamente buenos o, en su defecto, pobres. Al preguntarles por su actitud, esta es favorable hacia el cuidado en cifras similares al estudio realizado por Chellan et al..$^{16}$. En cambio, el hallazgo más interesante es el contraste con el nivel de comportamiento en la práctica de autocuidado, que en la mitad de la muestra es de carácter medio, repartiendo en partes iguales la buena práctica con la escasa práctica de autocuidado. Las personas ingresadas o sus cuidadores tienen conocimiento y actitud para el cuidado en la prevención del pie diabético como complicación de la diabetes, pero esos datos no se ven 
Tabla 3. Relación de los datos sociodemográficos y las variables principales del cuestionario KAP (Knowledge-Attitude-Practice)

\begin{tabular}{|c|c|c|c|c|c|c|c|c|c|c|c|}
\hline & \multicolumn{4}{|c|}{ Conocimientos n (\%) } & \multicolumn{3}{|c|}{ Actitud n (\%) } & \multicolumn{4}{|c|}{ Práctica en el autocuidado del pie diabético n (\%) } \\
\hline & Pobres & Medios & Buenos & $\begin{array}{l}\text { Valor } \\
\text { de } p\end{array}$ & $\begin{array}{c}\text { Poco } \\
\text { favorable }\end{array}$ & Favorable & $\begin{array}{l}\text { Valor } \\
\text { de } p\end{array}$ & Escasa & Media & Buena & $\begin{array}{l}\text { Valor } \\
\text { de } p\end{array}$ \\
\hline \multicolumn{12}{|l|}{ Edad } \\
\hline 30-69 años & $3(3,9)$ & $48(62,3)$ & $26(33,8)$ & \multirow{2}{*}{0,296} & $2(2,6)$ & $74(97,4)$ & \multirow{2}{*}{0,045} & $18(23,4)$ & $41(53,2)$ & $18(23,4)$ & \multirow{2}{*}{0,910} \\
\hline$<70$ años & $9(9,7)$ & $51(51,5)$ & $33(35,5)$ & & $10(10,5)$ & $85(89,5)$ & & $23(24,5)$ & $47(50)$ & $24(25,5)$ & \\
\hline \multicolumn{12}{|l|}{ Género } \\
\hline Masculino & $9(6,9)$ & $75(57,3)$ & $47(35,9)$ & \multirow{2}{*}{0,840} & $8(6,1)$ & $124(93,9)$ & \multirow{2}{*}{0,473} & $35(26,5)$ & $65(49,2)$ & $32(24,2)$ & \multirow{2}{*}{0,344} \\
\hline Femenino & $3(7,7)$ & $24(61,5)$ & $12(30,8)$ & & $4(10,3)$ & $35(89,7)$ & & $6(15,4)$ & $23(59)$ & $10(25,6)$ & \\
\hline \multicolumn{12}{|c|}{ Nivel de estudios } \\
\hline Sin estudios & $2(7,4)$ & $15(55,6)$ & $10(37)$ & \multirow{4}{*}{0,862} & $5(17,9)$ & $23(82,1)$ & \multirow{4}{*}{0,105} & $8(28,6)$ & $13(46,4)$ & $7(25)$ & \multirow{4}{*}{0,561} \\
\hline Primaria & $6(6,9)$ & $54(62,1)$ & $15(45,5)$ & & $4(4,5)$ & $84(95,5)$ & & $20(23)$ & $48(55,2)$ & $19(21,2)$ & \\
\hline Secundaria & $2(6,1)$ & $16(48,5)$ & $15(45,5)$ & & $2(6,3)$ & $30(93,8)$ & & $10(30,3)$ & $16(48,8)$ & $7(21,2)$ & \\
\hline Superiores & $2(8,7)$ & $14(60,9)$ & $7(30,4)$ & & $1(4,3)$ & $22(95,7)$ & & $3(13)$ & $11(47,8)$ & $9(39,1)$ & \\
\hline \multicolumn{12}{|c|}{ Tiempo de evolución de la diabetes } \\
\hline$<15$ años & $9(10,8)$ & $48(57,8)$ & $26(31,3)$ & \multirow{2}{*}{0,147} & $3(3,6)$ & $80(96,4)$ & \multirow{2}{*}{0,091} & $24(28,9)$ & $40(48,2)$ & $19(22,9)$ & \multirow{2}{*}{0,340} \\
\hline$\geq 15$ años & $3(3,4)$ & $51(58,6)$ & $33(37,9)$ & & $9(10,2)$ & $79(89,8)$ & & $17(19,3)$ & $48(54,5)$ & $23(26,1)$ & \\
\hline
\end{tabular}

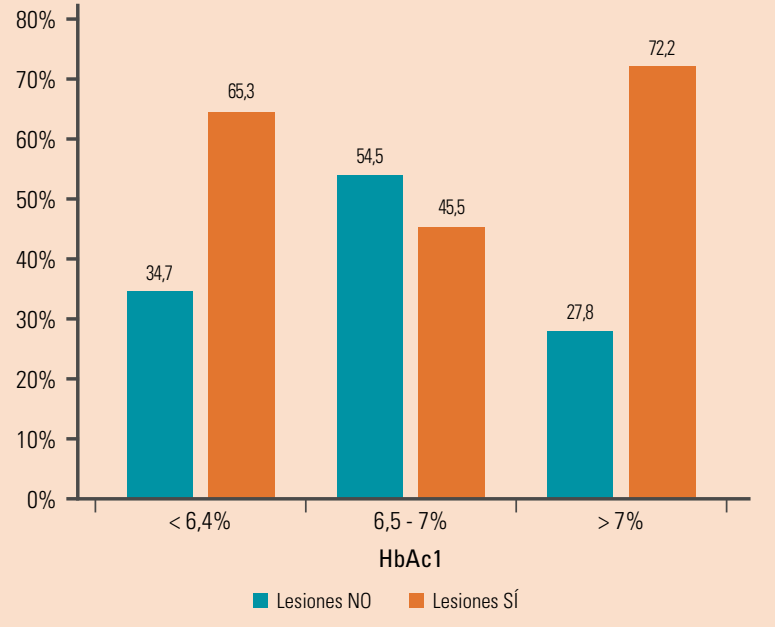

Figura 1. Relación de niveles de HbA1c y presencia de lesiones.

reflejados equitativamente en el comportamiento del autocuidado como práctica activa.

Asimismo, los resultados orientan sobre la necesidad de informar a las personas sobre el cuidado del pie diabético. Menos de la mitad de los encuestados refirieron haber sido informados, cifras que coinciden con estudios anteriores ${ }^{17}$. Sin embargo, a pesar de que no hay suficiente evidencia para afirmar que la educación por sí sola disminuye el riesgo de sufrir una úlcera en el pie, existe literatura científica que apoya el uso de seminarios de educación diabetológica sobre medidas preventivas y autocuidados como estrategias eficaces para mejorar conocimientos y hábitos adquiridos por el paciente ${ }^{18}$. La prevención es el primer paso para evitar complicaciones y reducir la incidencia de úlceras del pie.
La Asociación Española de Enfermería Vascular y Heridas (AEEV) considera imprescindible que en la educación sanitaria por los equipos de salud impliquen a los pacientes para que realicen la evaluación diaria de los pies, su hidratación, inspección del calzado antes de ponerlo o el corte de uńas horizontal entre otras.

Los resultados muestran una higiene diaria de los pies menor de la esperada. Sin obviar que existen diferencias socioculturales; estudios realizados en hospitales de Nigeria ${ }^{19}$, Malasia ${ }^{20}$ o Brasil ${ }^{21}$ muestran que la higiene de los pies diaria se realizó con menor frecuencia en nuestro entorno. En contraposición, la revisión de los pies en busca de heridas se llevó a cabo de forma similar que en el trabajo de Muhammad-Lutfi et al..$^{20} \mathrm{y}$ con más frecuencia que en el de Desalu et al. ${ }^{19}$ y Chiwanga ${ }^{17}$. La gran mayoría de los encuestados reconocen como forma correcta de cortar las uñas de los pies el corte recto. Aproximadamente un tercio de la población lo realiza de esta forma en un estudio similar ${ }^{19}$. Además, la revisión del calzado se realiza con más frecuencia que en estudios similares ${ }^{17,19,20,21}$.

El estudio evidencia que tanto las personas mayores de 70 años como menores de esta edad presentan actitud favorable al autocuidado; por lo tanto, consideramos que la edad no es un factor que influya en esta variante.

En lo referente a los niveles de HbAlc recogidos y la presencia de lesiones, a pesar de que esta relación haya salido significativa, los porcentajes de los niveles inferiores al 7\% presentan resultados invertidos entre ellos. Su explicación radica en que según Abdelhafiz y Sinclair ${ }^{22}$, los criterios para el diagnóstico de diabetes en personas de edad avanzada no son diferentes de los utilizados en la población más joven, pero coinciden con las recomendaciones del consenso español en que los objetivos a largo plazo se basan en concentraciones de HbAlc que debe ser del 7-7,5\% en personas mayores que viven en la comunidad y cuyo diagnóstico es reciente con función y cognición conservadas, sin complicaciones ni comorbilidades importantes y con buena expectativa de vida. Y en ancianos frágiles, con discapacidades, importantes complicaciones o comorbilidades y con expectativa de vida corta, los objetivos de 
control deben ser menos estrictos (HbA1c 7,6-8,5\%) $)^{22,23}$. Dada la alta prevalencia de diabetes en este grupo poblacional, se recomienda una valoración analítica anual que incluya $\mathrm{HbAl}_{1} \mathrm{c}^{24}$. Los objetivos de control metabólico deben ser individualizados, en función de las características diferenciales de cada paciente ${ }^{25}$.

En el desarrollo del estudio encontramos como limitaciones un sesgo de selección si tenemos presente que aquellos que quieran participar en el estudio sean los que más predispuestos estén en conocer su autocuidado y tengan, por esta razón, más conocimientos. Esto puede provocar que la muestra no sea representativa de la población, con lo que la interpretación de los resultados y emisión de conclusiones se realiza con cierta prudencia.
Además, existió un posible sesgo de medición a la hora de evaluar los conocimientos, actitudes y comportamientos de los participantes ante el autocuidado del pie diabético. Se intentó disminuir la probabilidad de este sesgo consensuando la utilización del cuestionario entre todos los miembros del equipo investigador.

En conclusión, este estudio ha objetivado que los usuarios de una unidad de cirugía vascular deben mejorar notablemente su comportamiento en autocuidado del pie diabético, así como mejorar su nivel de conocimiento en cuanto a esta complicación de la diabetes

\section{Conflicto de intereses}

Los autores declaran no tener ningún conflicto de intereses.

\section{Y BIBLIOGRAFÍA}

1. Matthew C. Riddle M, et al. ADA. Standards of medical care in Diabetes. Diabetes Care. 2018;41(Suppl 1):S13-27.

2. WHO. Informe mundial sobre la diabetes [Internet]. 2016. Disponible en: https://www.who.int/diabetes/global-report/es/

3. Aguayo A, Urrutia I, González Frutos T, Martínez R, Martínez-Indart $L$, Castaño $L$, et al. Prevalence of diabetes mellitus and impaired glucose metabolism in the adult population of the Basque Country, Spain. Diabet Med. 2017;34(5):662-6.

4. Prompers L, Schaper N, Apelqvist J, Edmonds M, Jude E, Mauricio $\mathrm{D}$, et al. Prediction of outcome in individuals with diabetic foot ulcers: Focus on the differences between individuals with and without peripheral arterial disease. The EURODIALE Study. Diabetologia. 2008;51(5):747-55

5. Faglia E, Clerici G, Scatena A, Caminiti M, Curci V, Prisco M, et al. Severity of demographic and clinical characteristics, revascularization feasibility, major amputation, and mortality rate in diabetic patients admitted to a tertiary diabetic foot center for critical limb ischemia: Comparison of 2 cohorts recruited at a 1. Ann Vasc Surg. 2014;28(7):1729-36.

6. Smeltzer S, Bare B, Hinkle J, Cheever K. Enfermería Médico-Quirúrgica. En: Brunner y Shudarth, eds. 12 a ed. Vol. 2 Philadelphia: Wolters Kluwer; 2013. p. 1236-8.

7. Zhang P, Lu J, Jing Y, Tang S, Zhu D, Bi Y. Global epidemiology of diabetic foot ulceration: a systematic review and meta-analysis. Ann Med. 2017;49(2):106-16.

8. Chadwick P, Edmonds M, MsCardle J, Armstrong D, Apelqvist J, Botros M, et al. Best Practice Guidelines: Wound Management in Diabetic Foot Ulcers. Vol. 5, Wounds International. 2013.

9. García Fernández FP, Soldevilla Agreda JJ, Torra i Bou JE. Atención Integral de las heridas crónicas. 2. ${ }^{\text {a }}$ ed. Fundacion Sergio Juán Jordán. GNEAUPP. 2016. 335 p.
10. Marinel.Io Roura J. Úlceras de la extrimidad inferior. 2. ${ }^{a}$ ed. Marinel.Io Roura J, editor. Barcelona; 2011.

11. Bakker K, Riley P. El año del pie diabético. Atención Sanit. 2005:50(1):11-4.

12. Ministerio de Salud. Grupo de trabajo de la Guía de Práctica Clínica sobre Diabetes tipo 2. Guía de Práctica Clínica sobre Diabetes tipo 2. Madrid: Plan Nacional para el Sistema Nacional de Salud del Ministerio de Sanidad y Consumo. Agencia de Evaluación de Tecnologías Sanitarias del País Vasco. 2008.

13. Gaspar Escayola JI. Programa de atención a enfermos crónicos dependientes. Gobierno de Aragón. Departamento de Salud y Consumo; 2004

14. NANDA International. Diagnósticos enfermeros. Definiciones y clasificación, 2015-2017. Herdman H, Kamitsuru S, editores. Barcelona: Elsevier; 2015. p. 472-4.

15. Aalaa M, Malazy T, Sanjari M, Peimani M, Mohajeri-Tehrani M. Nurses' role in diabetic foot prevention and care; a review. J Diabetes Metab Disord. 2012;11(1):24.

16. Chellan G, Srikumar S, Varma AK, Mangalanandan TS, Sundaram KR, Jayakumar RV, et al. Foot care practice - The key to prevent diabetic foot ulcers in India. Foot. 2012;22(4):298-302.

17. Chiwanga FS, Njelekela MA. Diabetic foot: prevalence, knowledge, and foot self-care practices among diabetic patients in Dar es Salaam, Tanzania-a cross-sectional study. J Foot Ankle Res [Internet]. 2015;8:20. Disponible en: https://www.ncbi.nlm.nih.gov/pmc/articles/PMC4462176/ pdf/13047_2015_Article_80.pdf

18. Dorresteijn JAN, Valk GD. Patient education for preventing diabetic foot ulceration. Diabetes Metab Res Rev. 2012;28:101-6.

19. Desalu 00, Salawu FK, Jimoh AK, Adekoya AO, Busari OA Olokoba AB. Diabetic foot care: self reported knowledge and practice among patients attending three tertiary hospital in Nigeria. Ghana Med J. 2011;45(2):60-5.

20. Muhammad-Lutfi AR, Zaraihah MR Anuar-Ramdhan IM. Knowledge and Practice of Diabetic Foot Care in an In- $\mathrm{Pa}$ tient Setting at a Tertiary Medical Center. Malaysian Orthop J. 2014;8(3):22-6

21. Natalia De Sá P, Moura JRA, Júnior EBDM, De Almeida PC De Macêdo SF, Da Silva ARV. Knowledge, attitudes and practices for the prevention of diabetic foot. Rev Gauch Enferm. 2014;35(3):36-42.

22. Abdelhafiz AH, Sinclair AJ. Diabetes in the elderly. Medicine (Baltimore) [Internet]. 2019;47(2):119-22. Disponible en: https://linkinghub.elsevier.com/retrieve/pii/ S1357303918302780

23. Formiga F, Gómez-Huelgas R, Rodríguez Mañas L. Características diferenciales de la diabetes mellitus tipo 2 en el paciente anciano. Papel de los inhibidores de la dipeptidil peptidasa 4. Rev Esp Geriatr Gerontol [Internet]. 2016;51(1):44-51. Disponible en: https://www.sciencedirect.com/science/article/pii/ S0211139X15000670

24. Sinclair A, Dunning T, Rodriguez-Mañas L. Diabetes in older people: new insights and remaining challenges. Lance Diabetes Endocrinol [Internet]. 2015;3(4):275-85. Disponible en: https://www.sciencedirect.com/science/article/abs/pii/ S2213858714701767

25. Inzucchi S, Bergenstal RM, Buse JB, Diamant M, Ferranini $E$, Nauck M, et al. Management of Hyperglycemia in Type 2 Diabetes, 2015: A Patient-Centered Approach. Diabetes Care [Internet]. 2015;(38):140-9. Disponible en: http://care.diabetesjournals.org/lookup/ 